\title{
Functional assessment of prefrontal lobes in patients with major depression disorder using a dual-mode technique of 3D-arterial spin labeling and ${ }^{18} \mathrm{~F}$-fluorodeoxyglucose positron emission tomography/computed tomography
}

\author{
CHANG FU $^{1}$, DAPENG SHI ${ }^{2}$, YONGJU GAO ${ }^{1}$ and JUNLING XU ${ }^{1}$ \\ Departments of ${ }^{1}$ Nuclear Medicine and ${ }^{2}$ Radiology, Zhengzhou University People's Hospital, \\ Zhengzhou, Henan 450000, P.R. China
}

Received October 23, 2015; Accepted February 24, 2017

DOI: $10.3892 /$ etm.2017.4594

\begin{abstract}
The aim of this study was to explore the functions of cerebral blood perfusion and glucose metabolism in the prefrontal lobe of patients with major depression disorder (MDD), and to analyze the correlations between these functional changes and depressive symptoms. 3D-arterial spin labeling (ASL) and ${ }^{18} \mathrm{~F}$-fluorodeoxyglucose (FDG) positron emission tomography/computed tomography (PET/CT) were successfully performed in 17 patients with MDD and 16 healthy controls in a resting state. The depressive symptoms of the patients were classified into seven factors and scored with the Hamilton Depression Rating Scale. Regional cerebral blood flow $(\mathrm{CBF})$ values and standardized uptake values (SUV) of ${ }^{18} \mathrm{~F}-\mathrm{FDG}$ in the whole brain were respectively compared between the patients and healthy controls using a two-sample t-test, and the correlations between the CBF and SUV in the prefrontal cerebral regions with the patients' Hamilton scores were evaluated using Pearson correlation analysis. Decreased regional $\mathrm{CBF}$ was indicated in the bilateral middle and the right superior frontal gyri, and decreased regional SUV was indicated in the bilateral superior, middle and inferior frontal gyri in the MDD patients compared with the controls. Positive correlations were observed between $\mathrm{CBF}$ values and aggregate Hamilton scores in the left middle and right middle frontal gyri of the patients. Positive correlations were also observed between SUVs and aggregate Hamilton scores in the left middle and right middle frontal gyri. ${ }^{18} \mathrm{~F}-\mathrm{FDG} \mathrm{PET} / \mathrm{CT}$ was indicated to be more sensitive than 3D-ASL in identifying the functional abnormalities in the prefrontal lobe. Decreased
\end{abstract}

Correspondence to: Dr Junling Xu, Department of Nuclear Medicine, Zhengzhou University People's Hospital, 7 Weiwu Road, Zhengzhou, Henan 450000, P.R. China

E-mail: episodede@sina.com

Key words: arterial spin labeling, cerebral blood flow, ${ }^{18}$ F-fluorodeoxyglucose, major depression disorder, positron emission tomography/computed tomography, standard uptake value
$\mathrm{CBF}$ and SUV in the prefrontal lobe were closely correlated with Hamilton score. The left middle frontal gyrus may be a key functional region in MDD.

\section{Introduction}

As a mental disorder, major depression disorder (MDD) is characterized by a significant and persistent depressed mood (1), accompanied by multiple cognitive and behavioral changes $(2,3)$. These behavioral changes are the result of cerebral dysfunction, which is based on molecular biological abnormalities in brain tissues. The prefrontal cortex is closely linked with many functions such as planning, cognition, memory, execution and executive control. Prefrontal dysfunction is often regarded as the pathological basis of depression onset and also as a sign of a depressive state (4). Investigating functional changes in the prefrontal lobe using imaging techniques, and analyzing correlations between the functional changes and clinical symptoms helps to elucidate the features of cerebral molecular biological abnormalities in depressive patients, and accurately locate the cerebral functional regions responsible for behavioral disorders. Consequently, this provides reliable markers for the clinical diagnosis and treatment of depression.

The 3D-arterial spin labeling (ASL) technique for magnetic resonance imaging and ${ }^{18} \mathrm{~F}$-fluorodeoxyglucose (FDG) positron emission tomography/computed tomography (PET/CT) can reflect functions of cerebral blood perfusion and glucose metabolism, respectively (5). Theoretically, the two functions should be consistent. Previous mono-mode imaging studies using ASL or ${ }^{18} \mathrm{~F}-\mathrm{FDG}$ PET have reported changes in cerebral blood perfusion and glucose metabolism in the prefrontal lobes of depressed patients $(6,7)$. A study conducted by Lui et al (7) indicated that, in non-refractory depressed patients, cerebral blood perfusion changes were mainly located in the bilateral frontal-cortical pathways. Lee et al (6) reported that glucose metabolism was reduced in the bilateral frontal cortices. Mayberg et al (8) reported that a reduction of cerebral ${ }^{18} \mathrm{~F}$-FDG standardized uptake values (SUVs) in the frontal cortex, particularly in the medial and dorsal frontal cortex, was a common manifestation in multiple types of 
depression. However, studies simultaneously assessing the functions of cerebral blood perfusion and glucose metabolism in depression patients using dual-mode imaging techniques have not yet been conducted.

Clinical depressive symptoms are usually evaluated using the Hamilton Depression Rating Scale (HAMD), which divides the depressive symptoms into seven factors (9): Anxiety, loss of weight, cognitive impairment, diurnal variation, retardation, insomnia and depressed mood. Functional changes of the frontal cortex in patients with MDD may have correlations with HAMD scores for each of the seven factors. Vasic et al (10) reported a positive correlation between depressive symptoms and cerebral blood flow (CBF) in the right middle frontal cortex. Graff-Guerrero et al (11) also indicated that the symptom factor scores of MDD were all positively or negatively correlated with the frontal cortical blood flow values. In a study that focused on the correlation between cerebral glucose metabolism and HAMD scores, positive correlations were observed in the inferior frontal and inferior orbital frontal gyrus, and negative correlations were observed in the dorsolateral prefrontal cortex (12). These results indicate that $\mathrm{CBF}$ and glucose metabolism in the frontal cortex of MDD patients may be correlated with depressive symptoms. In the current study, correlation between CBF values and SUVs in the prefrontal lobes, and the Hamilton scores of seven symptoms in MDD patients were analyzed in order to explore the clinical significance of these functional changes and accurately locate cerebral functional regions for depression.

Thus, 17 patients with MDD and 16 age- and gender-matched healthy controls underwent dual-mode imaging scans of brain 3D-ASL and ${ }^{18} \mathrm{~F}-\mathrm{FDG}$ PET/CT. The functions of the prefrontal lobes in the patients with MDD were then assessed and evaluated.

\section{Patients and methods}

Patient recruitment. A total of 23 patients with MDD were recruited from the psychological clinic of Zhengzhou University People's Hospital (Zhengzhou, China), from November 2012 to December 2013. A total of 18 age- and gender-matched healthy controls were recruited via advertisements. The clinical characteristics of the patient and control groups that were analyzed (after several exclusions) are detailed in Table I.

Patients were diagnosed by two experienced psychiatrists according to the criteria in the Diagnostic and Statistical Manual of Mental Disorders (4th edition) (13). Patients' depression and anxiety severity were assessed using HAMD and the Hamilton Anxiety Rating Scale (HAMA) (14), respectively. Depressive symptoms were classified into seven factors: Anxiety, weight, cognitive impairment, diurnal variation, retardation, sleep disturbance and hopelessness. All patients were diagnosed with MDD for the first time and had not received any antidepressant treatment prior to undergoing imaging examinations. All participants were selected using the following criteria: i) Right-handed; ii) 18-50 years old; iii) no history of neurological illness or other serious physical disease; and iv) no history of alcohol or drug dependence.

This study was approved by the Ethics Committee of Zhengzhou University People's Hospital. All subjects provided written informed consent.
Image acquisition. Brain ${ }^{18} \mathrm{~F}-\mathrm{FDG} \mathrm{PET} / \mathrm{CT}$ and 3D-ASL scans were carried out over a period of 3 days for the 23 patients and 18 healthy controls.

3D-ASL scans were acquired with a Discovery MR750 scanner (GE Healthcare Bio-Sciences, Pittsburgh, PA, USA). A 3D-fast spoiled gradient echo [repetition time (TR), 8.2 msec; echo time (TE), $3.2 \mathrm{msec}$; inversion time (TI), $450 \mathrm{msec}$; slice thickness, $1 \mathrm{~mm}$; 156 slices; image matrix, 256x256; field-of-view (FOV), 24x24 cm] and a 3D-ASL sequence (TR, 4,950 msec; TI, $450 \mathrm{msec}$; TE, $10.5 \mathrm{msec}$; slice thickness, 4 $\mathrm{mm}$; 36 slices; image matrix, 1024x8; FOV, 24x24 cm) were employed.

${ }^{18}$ F-FDG PET/CT images were obtained using a Discovery VCT PET-CT set (GE Healthcare Bio-Sciences). ${ }^{18} \mathrm{~F}-\mathrm{FDG}$ was compounded using a MINItrace medical cyclotron (GE Healthcare Bio-Sciences) and FDG automatic synthesis device. Prior to the examination, all patients were required to fast for $\geq 6 \mathrm{~h}$ and the fasting blood glucose level of the patients was required to be $<6.1 \mathrm{mmol} / 1$. ${ }^{18} \mathrm{~F}-\mathrm{FDG}$ was injected intravenously at a dose of $5.55 \mathrm{MBq} / \mathrm{kg}$. After the injection, subjects remained in a resting state in a quiet environment for a 50-min uptake period. The cerebral PET acquisition time was $10 \mathrm{~min}$. PET/CT scanning parameters were as follows: Voltage, $120 \mathrm{kV}$; current, $60 \mathrm{~mA}$; and thickness, $5 \mathrm{~mm}$. An acquisition counter using an iterative method was used to reconstruct transverse, sagittal and coronal images.

3D-ASL data analysis. Statistical parametric mapping (SPM8 software; Wellcome Trust Centre for Neuroimaging, London, $\mathrm{UK}$ ) was used to complete image preprocessing. First, CBF images were calculated from 3D-ASL images using the GE Advantage Workstation 4.5 (AW4.5; GE Healthcare Bio-Sciences). The CBF images were then converted into the Analyze7 format by SPM8. The converted images were spatially normalized to the space coordinates of the Talairach cerebral atlas. The normalized images were smoothed with an isotropic Gaussian kernel of $2 \times 2 \times 2 \mathrm{~mm}^{3}$ (full width at half maximum, FWHM). A target image with a voxel size of $2 \times 2 \times 2 \mathrm{~mm}^{3}$ and an image matrix of $128 \times 128$ was then prepared.

PET data analysis. Cerebral PET images were converted into the Analyze7 format using SPM8. The images were then spatially normalized to the space coordinates of the Talairach cerebral atlas. The normalized images were smoothed with an isotropic Gaussian kernel of $2 \times 2 \times 2 \mathrm{~mm}^{3}$ (FWHM). A target image with a voxel size of $2 \times 2 \times 2 \mathrm{~mm}^{3}$ and an image matrix of $128 \times 128$ was then prepared.

Statistical analysis. To compare CBF between MDD patients and controls, a second-level random-effects two-sample t-test was performed on individual CBF maps in a voxel-by-voxel manner. The resulting statistical map was set at a combined threshold of corrected $\mathrm{P}<0.005$ and a minimum cluster size of 10 voxels. Each cerebral region in which the $\mathrm{CBF}$ changed in MDD patients, compared with the control group, was used as a region of interest (ROI). The sketching of ROIs and the extraction of average $\mathrm{CBF}$ were performed using the AW4.5 workstation.

To compare SUV between MDD patients and controls, a second-level random-effects two-sample t-test of voxel-based 
Table I. Clinical and demographic characteristics of the patients with MDD and healthy controls.

\begin{tabular}{lccr}
\hline Characteristic & MDD $(\mathrm{n}=17)$ & Healthy control $(\mathrm{n}=16)$ & P-value \\
\hline Age (years) & $33.00 \pm 7.59$ & $34.59 \pm 8.96$ & $0.57^{\mathrm{a}}$ \\
Education (years) & $17.21 \pm 2.42$ & $16.95 \pm 1.84$ & $0.53^{\mathrm{a}}$ \\
Male/female (n/n) & $6 / 11$ & $5 / 11$ & $0.59^{\mathrm{b}}$ \\
HAMD & $19.50 \pm 2.57$ & $4.65 \pm 1.58$ & $<0.05^{\mathrm{a}}$ \\
HAMA & $12.49 \pm 4.12$ & $2.51 \pm 1.24$ & $<0.05^{\mathrm{a}}$
\end{tabular}

Values are presented as the mean \pm standard deviation. HAMA, Hamilton Anxiety Rating Scale; HAMD, Hamilton Depression Rating Scale; MDD, major depressive disorder. ${ }^{a}$ Determined by independent $t$-test; ${ }^{b}$ determined by $\chi^{2}$ test.

statistical analyses was performed on the cerebral ${ }^{18} \mathrm{~F}-\mathrm{FDG}$ PET images. Corrected $\mathrm{P}<0.005$ and a minimum cluster size of 10 voxels was used. Each cerebral region in which the SUV changed in MDD patients, compared with the control group, was used as an ROI. The sketching of ROIs and the calculation of average SUV were performed using the AW4.5 workstation.

Pearson correlation analysis was used to compare regional CBF values and SUV in abnormal activation cerebral regions of prefrontal lobes in MDD patients. It was also used to compare CBD and SUV in abnormal activation cerebral regions with HAMD scores. Analyses were conducted using SPSS 17.0 software (SPSS, Inc., Chicago, IL, USA). P<0.05 was considered to indicate a statistically significant difference.

\section{Results}

Patient eligibility. Following exclusion of 6 of the 23 MDD patients and 2 of the 18 healthy subjects due to excessive movement during 3D-ASL scanning, 17 patients and 16 healthy controls were included in the qualitative analyses (Table I). No significant differences were identified between the groups in terms of age, years of education or male/female ratio. However, HAMD and HAMA scores were significantly higher in the MDD group compared with the control group (both $\mathrm{P}<0.05$ ). The HAMD scores of the MDD patients and healthy controls are presented in greater detail in Table II.

Brain 3D-ASL and ${ }^{18} F-F D G$ PET results. Decreased CBF was demonstrated in the bilateral middle and the right superior frontal gyrus in patients with MDD compared with controls (Fig. 1 and Table III). No other regions with decreased or increased CBF were observed.

Decreased SUVs of ${ }^{18} \mathrm{~F}-\mathrm{PDG}$ were observed in the bilateral superior, middle and inferior frontal gyrus, bilateral superior and middle temporal gyrus, bilateral anterior cingulate cortex, bilateral putamen and caudate, and the left pallidum in patients with MDD compared with controls (Fig. 2 and Table III). Moreover, increased SUVs were observed in the bilateral hippocampus and left thalamus (Fig. 3 and Table III).

In the prefrontal cerebral regions, $\mathrm{CBF}$ values and SUV were positively correlated in the left middle frontal gyrus (Table III).

Analysis of the correlation of CBF and SUVs in prefrontal cerebral regions with HAMD scores. $\mathrm{CBF}$ values in the right
Table II. HAMD scores of patients with MDD and healthy controls.

\begin{tabular}{lrc}
\hline HAMD item & MDD & Control \\
\hline HAMD aggregate & $18.50 \pm 2.57$ & $4.65 \pm 1.58$ \\
Anxiety/somatization & $2.78 \pm 0.15$ & $0.65 \pm 0.02$ \\
Weight & $0.72 \pm 0.03$ & $0.31 \pm 0.03$ \\
Cognitive disturbance & $3.41 \pm 0.37$ & $0.78 \pm 0.05$ \\
Diurnal variation & $2.88 \pm 0.26$ & $0.62 \pm 0.03$ \\
Retardation & $3.63 \pm 0.45$ & $0.81 \pm 0.06$ \\
Sleep disturbance & $1.40 \pm 0.18$ & $0.56 \pm 0.02$ \\
Hopelessness & $3.68 \pm 0.54$ & $0.92 \pm 0.04$ \\
\hline
\end{tabular}

Values are presented as the mean \pm standard deviation. HAMD, Hamilton Depression Rating Scale; MDD, major depressive disorder.

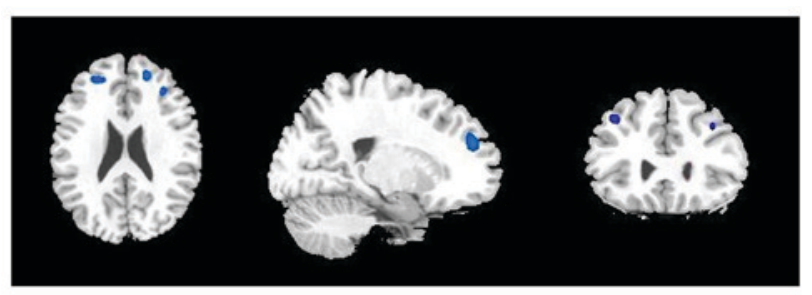

Figure 1. Cerebral regions (blue areas) with decreased regional blood flow in patients with major depression disorder compared with controls (cluster-level corrected $\mathrm{P}<0.005)$. Left to right: Transverse, sagittal and coronal $\mathrm{T} 1$ magnetic resonance imaging. The highlighted cerebral regions are the bilateral middle and the right superior frontal gyrus.

middle frontal gyrus were positively correlated with cognitive impairment. CBF values in the left middle frontal gyrus were negatively correlated with anxiety. CBF values in the left and right middle frontal gyrus were positively correlated with HAMD aggregate score (Table IV).

SUVs in the left and right middle frontal gyrus were positively correlated with cognitive impairment. SUVs in the right inferior frontal gyrus were negatively correlated with anxiety, and SUVs in the left middle and right inferior frontal gyrus were negatively correlated with retardation. Furthermore, the values in both the left and right middle frontal gyrus were positively correlated with HAMD aggregate score (Table V). 
Table III. Correlation ( $\mathrm{r}$ ) between SUVs and CBF values in the activated cerebral regions in the frontal lobes of patients with major depressive disorder.

\begin{tabular}{lccc}
\hline Cerebral area & SUV & CBF (ml/min/100 g) & \\
\hline Left superior frontal gyrus & $4.15 \pm 0.23$ & & \\
Left middle frontal gyrus & $4.72 \pm 0.56$ & $43.15 \pm 4.21$ & \\
Left inferior frontal gyrus & $4.29 \pm 0.41$ & $46.71 \pm 3.57$ \\
Right superior frontal gyrus & $4.39 \pm 0.35$ & $49.35 \pm 4.15$ & 0.12 \\
Right middle frontal gyrus & $4.92 \pm 0.63$ & & 0.04 \\
Right inferior frontal gyrus & $4.85 \pm 0.55$ & & \\
\hline
\end{tabular}

SUVs and CBF values are presented as the mean \pm standard deviation. SUV, standardized uptake values; $\mathrm{CBF}$, cerebral blood flow. ${ }^{\mathrm{a} C o r r e l a t i o n}$ is significant at the $\mathrm{P}$-value $<0.05$ level.
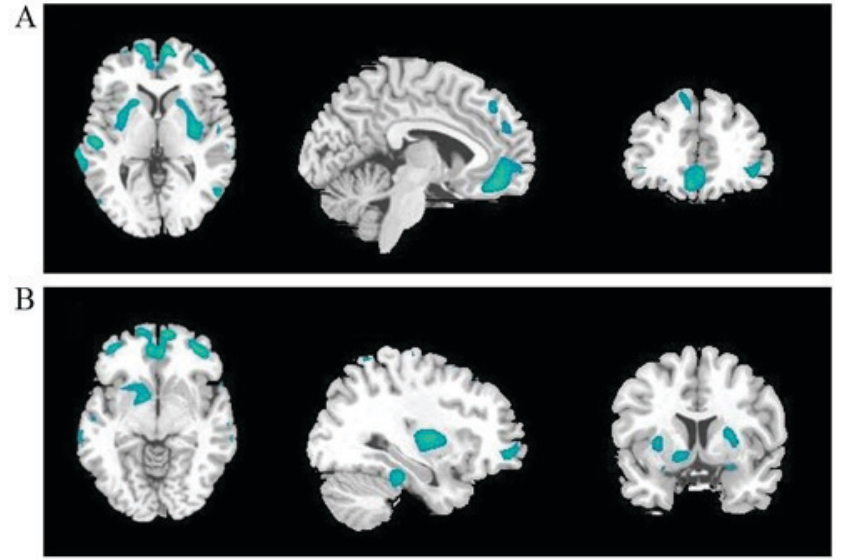

Figure 2. Cerebral regions (green areas) with decreased regional glucose metabolism in patients with major depression disorder compared with controls (cluster-level corrected $\mathrm{P}<0.005$ ). Left to right: Transverse, sagittal and coronal T1 magnetic resonance imaging. The highlighted cerebral regions are (A) the bilateral superior, middle and inferior frontal gyrus, bilateral superior and middle temporal gyrus, bilateral anterior cingulate cortex, bilateral putamen (B) the bilateral superior, middle and inferior frontal gyrus, bilateral anterior cingulate cortex, bilateral caudate, and the left pallidum.

\section{Discussion}

In the current study, patients with MDD had decreased cerebral blood perfusion and glucose metabolism in the prefrontal lobes compared with healthy controls. Although these findings are similar to those from previous mono-mode studies using ASL or FDG PET $(15,16)$, it is notable that the abnormal cerebral regions demonstrated in the two previous imaging studies did not fully correspond with those in this dual-mode trial. The cerebral hypometabolism in the FDG PET images involved a much wider brain area compared with the cerebral hypoperfusion images. Besides the bilateral superior, middle and inferior frontal gyrus, decreased SUVs were also observed in other cerebral regions, such as the bilateral middle and superior temporal gyrus, the bilateral lenticular nuclei and caudate nuclei, the left globus pallidus and the bilateral anterior cingulate gyri. Moreover, the bilateral hippocampi and the left thalamus presented hypermetabolism. Abnormalities in limbic-cortex-striatum-pallidus-thalamus (LCSPT) serve an important role in emotion regulation and conduction (17).

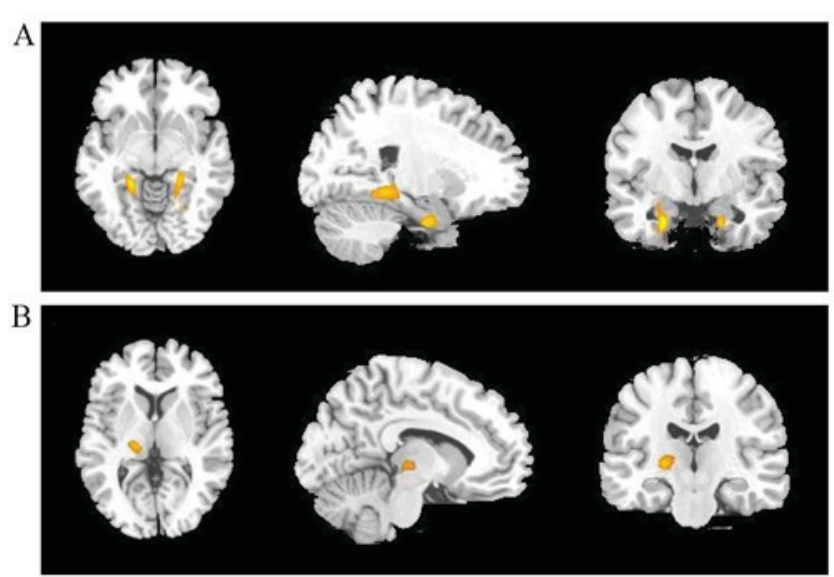

Figure 3. Cerebral regions (yellow areas) with increased regional glucose metabolism in patients with major depression disorder compared with controls (cluster-level corrected $\mathrm{P}<0.005$ ). Left to right: Transverse, sagittal and coronal $\mathrm{T} 1$ magnetic resonance imaging. Th highlighted cerebral regions are (A) the bilateral hippocampus and (B) the left thalamus.

Research has demonstrated that there are qualitative changes of LCSPT in patients with depression (18). The prefrontal cortex, anterior cingulate gyrus, basal ganglia, thalamus, hippocampus are important components of LCSPT (19). In the present study, abnormalities were identified in these cerebral regions, which were consistent with the currently recognized hypothesis of LCSPT neural circuitry. However, cerebral hypoperfusion in the brain 3D-ASL maps was only observed in the bilateral middle frontal and the right superior gyrus in these patients. The difference in the cerebral changes between the two methods may be associated with molecular biological changes of the brain in depression disorder (20) and also may result from the different imaging mechanisms used in the two techniques $(21,22)$. The current findings from dual-mode imaging not only imply that FDG PET is more sensitive than 3D-ASL in identifying functional abnormalities, but also suggest that the prefrontal lobe may be the principal brain region involved in depression. This lends support to the theory that prefrontal dysfunction is the pathological basis of depression onset and a sign of depressive status (4). SUVs or CBF values may be used as valid markers to quantitatively evaluate severity and monitor the effects of treatment in MDD. 
Table IV. Correlation (r) between HAMD scores and cerebral blood flow values in the abnormally activated cerebral regions of patients with major depressive disorder.

\begin{tabular}{lccc}
\hline HAMD item & $\begin{array}{c}\text { Left middle } \\
\text { frontal gyrus }\end{array}$ & $\begin{array}{c}\text { Right superior } \\
\text { frontal gyrus }\end{array}$ & $\begin{array}{c}\text { Right middle } \\
\text { frontal gyrus }\end{array}$ \\
\hline HAMD aggregate & $0.61^{\mathrm{a}}$ & 0.16 & $0.54^{\mathrm{a}}$ \\
Anxiety & $-0.69^{\mathrm{a}}$ & 0.28 & 0.13 \\
Weight & 0.26 & 0.17 & 0.42 \\
Cognitive impairment & 0.13 & -0.15 & $0.59^{\mathrm{a}}$ \\
Diurnal variation & -0.32 & -0.06 & 0.26 \\
Retardation & 0.31 & 0.28 & 0.27 \\
Sleep disturbance & 0.42 & 0.19 & 0.34 \\
Hopelessness & 0.19 & 0.46 & 0.33 \\
\hline
\end{tabular}

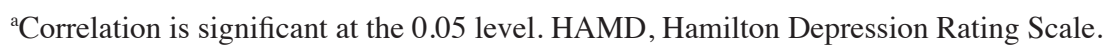

Table V. Correlation (r) between HAMD scores and standardized uptake values in the abnormally activated cerebral regions of patients with major depressive disorder.

\begin{tabular}{|c|c|c|c|c|c|c|}
\hline HAMD item & $\begin{array}{l}\text { Left superior } \\
\text { frontal gyrus }\end{array}$ & $\begin{array}{l}\text { Left middle } \\
\text { frontal gyrus }\end{array}$ & $\begin{array}{l}\text { Left inferior } \\
\text { frontal gyrus }\end{array}$ & $\begin{array}{l}\text { Right superior } \\
\text { frontal gyrus }\end{array}$ & $\begin{array}{l}\text { Right middle } \\
\text { frontal gyrus }\end{array}$ & $\begin{array}{l}\text { Right inferior } \\
\text { frontal gyrus }\end{array}$ \\
\hline HAMD aggregate & 0.09 & $0.58^{\mathrm{a}}$ & 0.13 & 0.16 & $0.62^{\mathrm{a}}$ & 0.23 \\
\hline Anxiety & 0.42 & 0.31 & 0.37 & 0.41 & 0.44 & $-0.71^{\mathrm{a}}$ \\
\hline Weight & 0.16 & -0.07 & 0.34 & 0.41 & 0.29 & 0.33 \\
\hline Cognitive impairment & -0.12 & $0.55^{\mathrm{a}}$ & 0.07 & 0.25 & $0.52^{\mathrm{a}}$ & 0.36 \\
\hline Diurnal variation & 0.05 & 0.23 & 0.12 & 0.35 & 0.14 & 0.29 \\
\hline Retardation & 0.37 & $-0.67^{a}$ & 0.03 & 0.16 & 0.23 & $-0.64^{\mathrm{a}}$ \\
\hline Sleep disturbance & -0.21 & 0.28 & -0.24 & 0.14 & 0.25 & 0.16 \\
\hline Hopelessness & 0.14 & -0.19 & 0.26 & 0.33 & 0.18 & 0.26 \\
\hline
\end{tabular}

${ }^{a}$ Correlation is significant at the 0.05 level. HAMD, Hamilton Depression Rating Scale.

Prefrontal dysfunction can directly interfere with the functional connectivity of default networks in depression (23), which results in relevant clinical symptoms (24). Therefore, functional abnormalities of the prefrontal lobe can be associated with multiple symptoms in patients with depression. In the current study, patients' clinical symptoms were divided into seven factors and quantitatively evaluated according to the HAMD. Correlation analysis between SUV and $\mathrm{CBF}$ values in each prefrontal gyrus and each symptom factor score were performed, in order to verify the function of each cerebral region and identify the cerebral region responsible for depression onset. Correlations were observed between the SUV and CBF values and multiple HAMD factor scores, including cognitive impairment, retardation, anxiety and aggregate scores. Correlations were primarily observed in the left middle, right middle and right inferior frontal gyrus. This implies that functional abnormality in the prefrontal lobe is closely associated with behavioral changes, and the bilateral middle and right inferior frontal gyrus may be the cerebral region responsible for depression onset in patients with MDD.
Changes in CBF values and SUV were most evident in the left middle frontal gyrus among all of the abnormal prefrontal regions. Not only were SUV and CBF values positively correlated with each other in this region, they were also closely correlated with multiple symptom factors. Such predominance of functional impairment in the left prefrontal lobe over the right side has also been reported in previous studies (25-27). Hamilton et al (25) reported that the local cerebral functional consistency in bilateral middle frontal gyri was negatively correlated with all seven HAMD factors. Nishi et al (26) reported that the local $\mathrm{CBF}$ in the left superior and right middle frontal gyrus in depressed patients at the resting state was positively correlated with cognitive impairment factor scores. These findings suggest that, among these prefrontal regions, the left middle frontal gyrus may be a key responsible functional region in patients with MDD. Although the current results from dual-mode imaging provide compelling evidence in support of this, a convincing theoretical explanation has not yet been reached on asymmetric prefrontal damage. It is proposed that the left prefrontal lobe may have more active functional connectivity in the default network of depression. This phenomenon of 
left-tendency is worthy of further attention in future research. Further studies on neural functional connectivity networks of the prefrontal lobe are required to elucidate this issue.

Besides the prefrontal lobes, there were other abnormal cerebral regions observed in PET images, such as decreased SUVs in the bilateral temporal lobes, bilateral basal ganglia and bilateral anterior cingulate gyri, and increased SUVs in the bilateral hippocampi and left thalamus. These findings are consistent with previous results (28). Kennedy et al (29) reported reduced glucose metabolism in the caudate nucleus and putamen in patients with depression. Kimbrell et al (30) detected decreased SUVs in anterior cingulated gyri. However, some contradicting results have also been reported. De Asis et al (31) reported increased FDG uptake in the anterior cingulate cortex. These contradictory results may result from differences in the types and stages of depression, and in treatment and imaging equipment. They may also reflect the complexity of the etiology and pathomechanisms of depressive disorder. Nevertheless, it may be noted that these abnormal cerebral regions are principal components of the neural circuit of depression, and can contribute to depression onset by disturbing the function of neural networks.

There were some limitations in the current study. First, the ages of the patients with MDD varied widely (18-50 years) and cerebral function, particularly FDG metabolism, may be affected by age. However, the age-matched healthy controls may have eliminated this effect. Second, the current study focused on assessing the function of the prefrontal lobe, so data obtained from other abnormally activated cerebral regions were not statistically analyzed or considered in-depth. Third, it was not possible to conduct a study contrasting pre- and post-therapy results for these patients, because only a small number of patients underwent dual-mode examinations after treatment. In the future, it would be valuable to conduct studies that overcome these limitations.

To the best of our knowledge, the current study was the first to use dual-mode 3D-ASL and FDG PET/CT to assess the function of the prefrontal lobe in MDD patients. The results from this trial suggest that ${ }^{18} \mathrm{~F}$-FDG PET/CT is more sensitive than 3D-ASL in identifying functional abnormalities in MDD patients. Decreased CBF and SUV in the prefrontal lobe were indicated to be closely correlated with depressive symptoms in MDD patients. The left middle frontal gyrus may be a key responsible functional region in MDD.

\section{Acknowledgements}

The authors would like to thank Weifeng Zhang, Huiqiang Li, Min Guan and Li Zhang for their assistance in patient scanning and data analysis.

\section{References}

1. Knutson B, Bhanji JP, Cooney RE, Atlas LY and Gotlib IH: Neural responses to monetary incentives in major depression. Biol Psychiatry 63: 686-692, 2008.

2. Adrien J: Neurobiological bases for the relation between sleep and depression. Sleep Med Rev 6: 341-351, 2002.

3. Macey PM, Woo MA, Kumar R, Cross RL and Harper RM: Relationship between obstructive sleep apnea severity and sleep, depression and anxiety symptoms in newly-diagnosed patients. PLoS One 5: e10211, 2010.
4. Kameyama M, Fukuda M, Yamagishi Y, Sato T, Uehara T, Ito M, Suto T and Mikuni M: Frontal lobe function in bipolar disorder: A multichannel near-infrared spectroscopy study. Neuroimage 29: 172-184, 2006.

5. Boscolo Galazzo I, Mattoli MV, Pizzini FB, De Vita E, Barnes A, Duncan JS, Jäger HR, Golay X, Bomanji JB, Koepp M, et al: Cerebral metabolism and perfusion in MR-negative individuals with refractory focal epilepsy assessed by simultaneous acquisition of (18)F-FDG PET and arterial spin labeling. Neuroimage Clin 11: 648-657, 2016.

6. Lee HS, Choo IH, Lee DY, Kim JW, Seo EH, Kim SG, Park SY, Shin JH, Kim KW and Woo JI: Frontal dysfunction underlies depression in mild cognitive impairment: A FDG-PET study. Psychiatry Investig 7: 208-214, 2010.

7. Lui S, Parkes LM, Huang X, Zou K, Chan RC, Yang H, Zou L, Li D, Tang H, Zhang T, et al: Depressive disorders: Focally altered cerebral perfusion measured with arterial spin-labeling MR imaging. Radiology 251: 476-484, 2009.

8. Mayberg HS, Brannan SK, Tekell JL, Silva JA, Mahurin RK, McGinnis S and Jerabek PA: Regional metabolic effects of fluoxetine in major depression: Serial changes and relationship to clinical response. Biol Psychiatry 48: 830-843, 2000.

9. Hamilton M: A rating scale for depression. J Neurol Neurosurg Psychiatry 23: 56-62, 1960.

10. Vasic N, Wolf ND, Grön G, Sosic-Vasic Z, Connemann BJ, Sambataro F, von Strombeck A, Lang D, Otte S, Dudek M and Wolf RC: Baseline brain perfusion and brain structure in patients with major depression: A multimodal magnetic resonance imaging study. J Psychiatry Neurosci 40: 412-421, 2015.

11. Graff-Guerrero A, González-Olvera J, Mendoza-Espinosa Y, Vaugier V and García-Reyna JC: Correlation between cerebral blood flow and items of the Hamilton rating scale for depression in antidepressant-naive patients. J Affect Disord 80: 55-63, 2004

12. Wu JC, Gillin JC, Buchsbaum MS, Schachat C, Darnall LA, Keator DB, Fallon JH and Bunney WE: Sleep deprivation PET correlations of Hamilton symptom improvement ratings with changes in relative glucose metabolism in patients with depression. J Affect Disord 107: 181-186, 2008.

13. American Psychiatric Association: Diagnostic and statistical manual of mental disorders. 4th edition. Washington, DC, 2000.

14. Maier W, Buller R, Philipp M and Heuser I: The Hamilton anxiety scale: Reliability, validity and sensitivity to change in anxiety and depressive disorders. J Affect Disord 14: 61-68, 1988.

15. Orosz A, Jann K, Federspiel A, Horn H, Höfle O, Dierks T, Wiest R, Strik W, Müller T and Walther S: Reduced cerebral blood flow within the default-mode network and within total gray matter in major depression. Brain Connect 2: 303-310, 2012.

16. Wei K, Xue HL, Guan YH, Zuo CT, Ge JJ, Zhang HY, Liu BJ, Cao YX, Dong JC and Du YJ: Analysis of glucose metabolism of (18)F-FDG in major depression patients using PET imaging: Correlation of salivary cortisol and $\alpha$-amylase. Neurosci Lett 629: 52-57, 2016.

17. Clark L, Chamberlain SR and Sahakian BJ: Neurocognitive mechanisms in depression: Implications for treatment. Annu Rev Neurosci 32: 57-74, 2009.

18. Seminowicz D, Mayberg H, McIntosh A, Goldapple K, Kennedy S, Segal Z and Rafi-Tari S: Limbic-frontal circuitry in major depression: A path modeling metanalysis. Neuroimage 22: 409-418, 2004.

19. Nauta WJ: Neural associations of the frontal cortex. Acta Neurobiol Exp (Wars) 32: 125-140, 1972.

20. van Hoeij F, Keijsers R, Loffeld B, Dun G, Stadhouders P and Weusten B: Incidental colonic focal FDG uptake on PET/CT: Can the maximum standardized uptake value (SUVmax) guide us in the timing of colonoscopy? Eur J Nucl Med Mol Imaging 42: 66-71, 2015.

21. Vidorreta M, Wang Z, Rodríguez I, Pastor MA, Detre JA and Fernández-Seara MA: Comparison of 2D and 3D single-shot ASL perfusion fMRI sequences. Neuroimage 66: 662-671, 2013.

22. Towgood KJ, Pitkanen M, Kulasegaram R, Fradera A, Soni S, Sibtain N, Reed LJ, Bradbeer C, Barker GJ, Dunn JT, et al: Regional cerebral blood flow and FDG uptake in asymptomatic HIV-1 men. Hum Brain Mapp 34: 2484-2493, 2013.

23. Greicius MD, Flores BH, Menon V, Glover GH, Solvason HB, Kenna H, Reiss AL and Schatzberg AF: Resting-state functional connectivity in major depression: Abnormally increased contributions from subgenual cingulate cortex and thalamus. Biol Psychiatry 62: 429-437, 2007. 
24. Lui S, Deng W, Huang X, Jiang L, Ma X, Chen H, Zhang T, Li X, Li D, Zou L, et al: Association of cerebral deficits with clinical symptoms in antipsychotic-naive first-episode schizophrenia: An optimized voxel-based morphometry and resting state functional connectivity study. Am J Psychiatry 166: 196-205, 2009.

25. Hamilton JP, Etkin A, Furman DJ, Lemus MG, Johnson RF and Gotlib IH: Functional neuroimaging of major depressive disorder: A meta-analysis and new integration of base line activation and neural response data. Am J Psychiatry 169: 693-703, 2012.

26. Nishi H, Sawamoto N, Namiki C, Yoshida H, Dinh HD, Ishizu K, Hashikawa K and Fukuyama H: Correlation between cognitive deficits and glucose hypometabolism in mild cognitive impairment. J Neuroimaging 20: 29-36, 2010.

27. Bench CJ, Friston KJ, Brown RG, Scott LC, Frackowiak RS and Dolan RJ: The anatomy of melancholia-focal abnormalities of cerebral blood flow in major depression. Psychol Med 22: 607-615, 1992.
28. Brody AL, Saxena S, Silverman DH, Alborzian S, Fairbanks LA, Phelps ME, Huang SC, Wu HM, Maidment K and Baxter LR Jr: Brain metabolic changes in major depressive disorder from pre-to post-treatment with paroxetine. Psychiatry Res 91: 127-139, 1999.

29. Kennedy SH, Evans KR, Krüger S, Mayberg HS, Meyer JH, McCann S, Arifuzzman AI, Houle S and Vaccarino FJ: Changes in regional brain glucose metabolism measured with positron emission tomography after paroxetine treatment of major depression. Am J Psychiatry 158: 899-905, 2001.

30. Kimbrell TA, Ketter TA, George MS, Little JT, Benson BE, Willis MW, Herscovitch P and Post RM: Regional cerebral glucose utilization in patients with a range of severities of unipolar depression. Biol Psychiatry 51: 237-252, 2002.

31. de Asis JM, Silbersweig DA, Pan H, Young RC and Stern E Neuroimaging studies of fronto-limbic dysfunction in geriatric depression. Clin Neurosci Res 2: 324-330, 2003. 